\title{
Serum Glycocholic Acid-to-Total Bile Acid Ratio Is Independently Associated with Nonalcoholic Fatty Liver Disease: A Retrospective Cross-Sectional Study
}

\author{
Hu Li, ${ }^{1}$ Jin Ma, ${ }^{2}$ Leilei Gu, ${ }^{3}$ Peizhan Chen, ${ }^{4}$ Li Chen $\mathbb{D}^{3},{ }^{3}$ and Xinxin Zhang $\mathbb{D}^{1,4}$ \\ ${ }^{1}$ Department of Infectious Disease, Research Laboratory of Clinical Virology, Ruijin Hospital, Shanghai Jiao Tong University, \\ School of Medicine, Shanghai 200025, China \\ ${ }^{2}$ Department of Physical Examination Center, Ruijin Hospital, Shanghai Jiao Tong University, School of Medicine, \\ Shanghai 201821, China \\ ${ }^{3}$ Department of Gastroenterology, Ruijin Hospital, Shanghai Jiao Tong University, School of Medicine, Shanghai 201821, China \\ ${ }^{4}$ Clinical Research Center, Ruijin Hospital, Shanghai Jiao Tong University, School of Medicine, Shanghai 201821, China \\ Correspondence should be addressed to Li Chen; drchenli@163.com and Xinxin Zhang; zhangx@shsmu.edu.cn
}

Received 25 December 2020; Revised 25 January 2021; Accepted 16 February 2021; Published 27 February 2021

Academic Editor: Udayan Apte

Copyright (C) $2021 \mathrm{Hu} \mathrm{Li}$ et al. This is an open access article distributed under the Creative Commons Attribution License, which permits unrestricted use, distribution, and reproduction in any medium, provided the original work is properly cited.

\begin{abstract}
Introduction and Aims. Bile acids play an essential role in the progression of nonalcoholic fatty liver disease (NAFLD). This study was aimed at investigating the association of the serum glycocholic acid- (GCA-) to-total bile acid (TBA) ratio with NAFLD in the general population. Materials and Methods. A total of 6708 subjects (2859 cases with NAFLD and 3849 controls) were enrolled in the development cohort and additional 1568 subjects (784 cases with NAFLD and 784 controls) in an independent validation cohort. Demographic characteristics and biochemical data were compared between subjects with NAFLD and controls. Multivariate logistic regression analysis was performed to determine the association of the GCA-to-TBA ratio with NAFLD. A novel model incorporating the GCA-to-TBA ratio was developed for screening NAFLD from the general population. Results. The serum TBA and GCA levels were significantly higher in subjects with NAFLD than in those without NAFLD (2.8 (2.0-4.2) $\mu \mathrm{mol} / \mathrm{L}$ vs. $2.5(1.8-3.7) \mu \mathrm{mol} / \mathrm{L}$ and $1.30(1.10-1.53) \mu \mathrm{g} / \mathrm{mL}$ vs. $1.28(1.08-1.50) \mu \mathrm{g} / \mathrm{mL}$, respectively, all $p \leq 0.01)$, whereas the serum GCA-to-TBA ratio was significantly lower in subjects with NAFLD than in subjects without NAFLD (0.44 (0.33-0.60) vs. $0.48(0.36-0.64), p \leq 0.01)$. Logistic regression analysis showed that the GCA-to-TBA ratio was independently associated with NAFLD after adjustment for confounding factors (odds ratio: $0.81,95 \%$ confidence interval (CI): $0.71-0.92, p \leq 0.01$ ). The area under the receiver operating characteristic curve of the novel developed GCA-to-TBA ratio score model in discriminating NAFLD was 0.84 (95\% CI: 0.83-0.85) in the development cohort and was 0.91 (95\% CI: 0.36-0.65) in the validation cohort. Conclusion. The serum GCA-to-TBA ratio is independently associated with NAFLD. A simple novel model incorporating the GCA-to-TBA ratio score has a good performance in discriminating NAFLD from the general population.
\end{abstract}

\section{Introduction}

Nonalcoholic fatty liver disease (NAFLD), a rapidly growing metabolic disease associated with type 2 diabetes mellitus and obesity, is the most common chronic liver disease worldwide [1]. Epidemiological studies have showed that the prevalence of NAFLD is above $20 \%$ in the general population [2] and reaches up to more than $50 \%$ in the diabetes population globally [3]. A cross-sectional study showed that the prevalence of
NAFLD in East China was 43.3\% in 2016 [4]. NAFLD is a heterogeneous disease comprising nonalcoholic fatty liver (NAFL), nonalcoholic steatohepatitis (NASH), fatty liverassociated cirrhosis, and hepatocellular carcinoma [5]. NASH, accounting for approximately $20 \%$ of NAFLD [6], is characterized by a varied degree of hepatocellular ballooning, necroinflammation, and fibrosis.

Over the past decade, an increasing number of studies have focused on bile acids (BAs) and their metabolism 
regulation. Serum total BAs (TBAs) comprise substantial amounts of primary BAs and secondary BAs. Primary BAs, including conjugated and nonconjugated BAs, are synthesized from cholesterol in hepatocytes via a series of enzyme-mediated oxidation reactions and then released into the intestine. In the intestine, primary BAs are deconjugated by gut microbiota to form secondary BAs. A large part of intestinal BAs is reabsorbed actively and recirculated to the liver through the portal vein. BAs not only facilitate absorption of lipids and lipid-soluble vitamins in the intestine but also regulate and maintain lipid and glucose homeostasis as signal molecules via the farnesoid $\mathrm{X}$ receptor (FXR) and Takeda G protein-coupled receptor 5 (TGR5) [7]. Therefore, $\mathrm{BA}$ receptors and related metabolic pathways have been potential therapeutic targets for NASH [8].

Previous studies demonstrated an increased level of serum TBA in patients with NAFLD compared with healthy control [9-11]. Moreover, the serum TBA levels increase with the development of liver fibrosis in patients with NAFLD. In terms of the BA profiles, the proportion of conjugated primary BAs increases, whereas that of unconjugated primary BAs significantly decreases with the progression of liver fibrosis [12]. Moreover, the specific changes in serum TBA are correlated with histological features of NASH, including higher grades of steatosis, lobular and portal inflammation, and hepatocyte ballooning [13]. These studies suggest that serum BA profiles may serve as a biomarker for identifying NASH and evaluating the severity or even pathological phenotype of NASH, but these findings need to be verified in a large cohort.

In a cross-sectional study with large sample sizes, Zhang et al. [14] showed that serum TBA levels were higher in patients with NAFLD than in those without NAFLD. However, multivariate logistic regression analysis showed no association between serum TBA and NAFLD after adjusting for confounding factors, which may be partly attributed to the diverse compositions of circulating TBAs. Glycocholic acid (GCA), a glycine-conjugated primary BA, is correlated with liver fibrosis $(F \geq 3)$ in patients with NAFLD [15], but its relationship with NAFLD in the general population remains unknown. In this study, we aimed to evaluate the association of serum GCA, TBA, and GCA-to-TBA ratio with NAFLD in a large population and determine whether the serum GCA-to-TBA ratio could be an indicator for NAFLD development.

\section{Materials and Methods}

2.1. Study Population. A total of 6708 subjects (2859 cases with NAFLD and 3849 controls) were enrolled in the development cohort at the Medical Examination Center of Ruijin Hospital, Shanghai Jiao Tong University, School of Medicine, during the period from January 2017 to December 2019. Additional 1568 subjects (784 cases with NAFLD and 784 controls) were enrolled in an independent validation cohort from January 2020 to June 2020. These subjects were scheduled to undergo a medical examination, including medical history, physical examination, serum biochemical tests, abdominal ultrasound examination, electrocardiography, and imaging examinations.
The following subjects were excluded from the study: (1) subjects less than 18 years of age; (2) those who had excessive alcohol consumption $(>30 \mathrm{~g} / \mathrm{d}$ for men and $>20 \mathrm{~g} / \mathrm{d}$ for women) [16]; (3) those who had a history of viral hepatitis, autoimmune hepatitis, or other known causes of chronic liver disease; (4) those who had hypothyroidism or polycystic ovarian syndrome; and (5) those having history of Chinese herbal medication and medications that induce liver injury, such as acetaminophen, within three months. This study was approved by the Institutional Ethics Committee of Shanghai Ruijin Hospital and complied with the Declaration of Helsinki.

2.2. Data Collection and Laboratory Assessments. Medical history, demographic characteristics (including age, sex, height, and body weight), laboratory data (including total blood cell counts and biochemical tests), and abdominal ultrasound and electrocardiography results were collected from all subjects meeting the above criteria at the time of enrolment. Blood samples were drawn from peripheral veins for total blood cell counts and biochemical tests after 8-12 h overnight fasting and then processed and measured at the Clinical Laboratory Diagnostic Center of Shanghai Ruijin Hospital, Shanghai Jiao Tong University School of Medicine. Serum biochemical tests were performed using an automatic biochemistry analyzer (AU5800 Automatic Biochemical Analyzer; Beckman Coulter, Inc., California, USA). Serum levels of GCA and TBA were measured using a cholylglycine assay kit (homogeneous enzyme immunoassay) (Evermed, Suzhou, China) and total bile acid assay kit (enzyme cycle method) (DiaSys, Shanghai, China), respectively. Other biochemical parameters were measured using commercially available reagents. Total blood cell counts were measured using an LH750 Automatic Blood Cell Analyzer (Beckman Coulter, Inc., California, USA). All laboratory tests were standardized and certified.

2.3. Definitions. NAFLD was diagnosed according to the American Association for the Study of Liver Diseases guidelines based on evidence of fatty liver by abdominal ultrasonography without significant alcohol consumption and other known chronic liver diseases [16]. A trained ultrasonographist who was blind to the clinical and laboratory data performed abdominal ultrasound examination for all enrolled subjects. Hypertension was diagnosed when blood pressure was $>140 / 90 \mathrm{mmHg}$ by more than two measurements or the subjects had a definite history of hypertension and treatment with antihypertensive drugs. Diabetes was diagnosed based on fasting serum glucose levels $\geq 7.0 \mathrm{mmol} / \mathrm{L}$ or $2 \mathrm{~h}$ postprandial serum glucose $\geq 11.1 \mathrm{mmol} / \mathrm{L}$, according to the 2019 diagnosis and management guidelines [17]. Patients who showed normal serum glucose but had a history of diabetes mellitus and treatment with hypoglycemic agents were also considered to have diabetes. Metabolic syndrome refers to subjects with three or more of the following features: (1) abdominal obesity with waist circumference $\geq 90 \mathrm{~cm}$ in men or $85 \mathrm{~cm}$ in women, (2) blood pressure value $\geq 130 / 85 \mathrm{mmHg}$ or treatment with antihypertension drugs, (3) fasting serum triglyceride level $\geq 1.7$ $\mathrm{mmol} / \mathrm{L}$ or treatment with lipid-lowering drugs, (4) fasting serum high-density lipoprotein cholesterol level $\leq 1.0 \mathrm{mmol} / \mathrm{L}$ 
in men or $1.3 \mathrm{mmol} / \mathrm{L}$ in women, and (5) fasting serum glucose $\geq 5.6 \mathrm{mmol} / \mathrm{L}$ or a history of type 2 diabetes mellitus. The body mass index (BMI) was calculated as body weight in kilograms divided by the square of height in meters.

2.4. Statistical Analysis. Categorical variables were expressed as frequency and percentages, and differences between groups were compared using Pearson's $x^{2}$ test. Continuous variables were expressed as median and interquartile range, and differences were compared using the Wilcoxon rank or KruskalWallis sum test. Multivariate logistic regression analysis was performed to determine the risk factors associated with NAFLD. The diagnostic performances of the GCA-to-TBA ratio score model, hepatic steatosis index (HSI) [18], and TyG triglyceride $\times$ glucose $(\mathrm{TyG})$ index [19] were analyzed by calculating the area under the receiver operating characteristic curve (AUROC) and 95\% confidence interval (CI). The TyG index was calculated as the $\mathrm{Ln}$ [fasting triglycerides (mg $/ \mathrm{dL}) \times$ fasting glucose $(\mathrm{mg} / \mathrm{dL}) / 2]$. The optimal diagnostic cutoff value was found by the Youden index. Sensitivity and specificity were calculated for the optimal cutoff point. The diagnostic performances of different models were compared using the DeLong test. All statistical analyses were conducted using R software (version 3.6.1, the R Project for Statistical Computing). The analysis of diagnostic performance was performed using the R package pROC (version 1.15.3) [20]. A two-tailed $p$ value $<0.05$ was considered statistically significant.

\section{Results}

3.1. Clinical Characteristics and Biochemical Data of the Enrolled Subjects in the Development Cohort. A total of 6708 subjects were enrolled in the development cohort. The general prevalence of NAFLD was $42.6 \%$ (2859/6708). The prevalence of NAFLD in males was higher than that in females $(50.7 \%$ vs. $28.5 \%, p \leq 0.01)$. The median age and BMI were 44 (36-54) years and $24.4(22.2-26.7) \mathrm{kg} / \mathrm{m}^{2}$, respectively. A total of $63.6 \%(4266 / 6708)$ of the subjects were male. The incidences of hypertension, diabetes, and metabolic syndrome were $26.0 \%$ (1744/6708), 5.2\% (347/6708), and 14.2\% (954/6708). Subjects with NAFLD had a significantly higher incidence of hypertension $(32 \%$ vs. $19.1 \%, p \leq 0.01)$, diabetes (9.1\% vs. $2.3 \%, p \leq 0.01)$, and metabolic syndrome $(25.6 \%$ vs. $5.7 \%, p \leq 0.01)$ than those without NAFLD. In addition, subjects with NAFLD tended to be older and had a higher BMI than those without NAFLD (47 (39-55) vs. 42 (35-52) years and 26.4 (24.7-28.3) vs. 22.9 (21.1-24.7) $\mathrm{kg} / \mathrm{m}^{2}$, respectively, all $\left.p \leq 0.01\right)$. As expected, serum alanine aminotransferase and aspartate aminotransferase levels were higher in subjects with NAFLD than in those without NAFLD. The comparison of other biochemical data between subjects with NAFLD and those without NAFLD is listed in Table 1.

3.2. Serum Levels of TBA and GCA Were Increased in NAFLD but Not Associated with NAFLD Development. As shown in Table 1, the median serum TBA levels of subjects with NAFLD were higher than those without NAFLD (2.8 (2.0-4.2) vs. 2.5
(1.8-3.7) $\mu \mathrm{mol} / \mathrm{L}, p \leq 0.01)$, and the serum GCA level of subjects with NAFLD was also higher than that of subjects without NAFLD (1.30 (1.10-1.53) vs. $1.28(1.08-1.50) \mu \mathrm{g} / \mathrm{mL}$, $p \leq 0.01)$. As serum TBA and GCA were influenced by some factors such as sex and age, multivariate logistic regression was used to determine whether the levels of serum TBA or GCA were independent factors associated with NAFLD development. After adjustment for age, sex, BMI, diabetes status, hypertension, and metabolic syndrome, the association between TBA or GCA and NAFLD was not significant $(p>0.05)$.

3.3. Incidence of NAFLD Increased with the Decrease in the Serum GCA-to-TBA Ratio. The serum GCA-to-TBA ratio of subjects with or without NAFLD was calculated. Interestingly, this ratio was significantly lower in subjects with NAFLD than in those without NAFLD $(0.44(0.33-0.60)$ vs. $0.48(0.36-0.64), p \leq 0.01)$. To better understand the relationship between the serum GCA-to-TBA ratio and the prevalence of NAFLD, all subjects were divided into four groups by quartiles of the GCA-to-TBA ratio. Quartile 1 (Q1) was the GCA-to-TBA ratio $<0.75$; quartile 2 (Q2), GCA-toTBA ratio 0.75-1.00; quartile 3 (Q3), GCA-to-TBA ratio 1.00-1.34; and quartile 4 (Q4), GCA-to-TBA ratio $\geq 1.34$. The incidence of NAFLD, metabolic syndrome, and diabetes and median values of biochemical parameters were calculated according to the quartiles of the GCA-to-TBA ratio. As shown in Figure 1, the incidence of NAFLD decreased with the increase in the GCA-to-TBA ratio ( $48.6 \%$ vs. $45.0 \%$ vs. $38.2 \%$ vs. $38.7 \%$, $p$ trend $\leq 0.01)$. Moreover, the same trend was observed with regard to the incidence of metabolic syndrome ( $16.8 \%$ vs. $14.5 \%$ vs. $13.7 \%$ vs. $12.1 \%, p$ trend $\leq 0.01$ ) and diabetes ( $6.9 \%$ vs. $5.6 \%$ vs. $4.5 \%$ vs. $3.7 \%$, $p$ trend $\leq 0.01)$. Biochemical data except for total cholesterol and low-density lipoprotein cholesterol also decreased with the increase in the GCA-to-TBA ratio $(p \leq 0.01)$ (Table 2 ).

3.4. Serum GCA-to-TBA Ratio Was Independently Associated with NAFLD Development. As serum GCA or TBA level was not an independent indicator for NAFLD development as demonstrated above, we investigated whether the GCA-toTBA ratio was associated with NAFLD development. As shown in Figure 2, multivariable logistic analysis suggested that the serum GCA-to-TBA ratio was independently associated with NAFLD development (odds ratio (OR): 0.81, 95\% confidence interval (CI): 0.71-0.92; $p \leq 0.01$ ) after adjustment for age, sex, BMI, hypertension, diabetes, and metabolic syndrome. We then performed a gender-stratified analysis to test the effect of the GCA-to-TBA ratio on NAFLD further. The serum GCA-to-TBA ratio was still independently associated with NAFLD development in both male (OR: 0.85, 95\% CI: $0.73-0.99, p=0.042$ ) and female (OR: $0.73,95 \%$ CI: 0.58 $0.92, p \leq 0.01)$ (Table 3).

3.5. The Value of the GCA-to-TBA Ratio in Discriminating NAFLD Subjects from the General Population. A ROC curve analysis was performed to evaluate whether the GCA-toTBA ratio could discriminate NAFLD subjects from the general population. The area under the receiver operating characteristic curve (AUC) of the GCA-to-TBA ratio was 
TABLE 1: Comparison of clinical characteristics and biochemical data between subjects with NAFLD and those without NAFLD in the development and validation cohort.

\begin{tabular}{|c|c|c|c|c|c|c|}
\hline & \multicolumn{2}{|c|}{ Development cohort } & \multirow[b]{2}{*}{$p$} & \multicolumn{2}{|c|}{ Validation cohort } & \multirow{2}{*}{$p$} \\
\hline & Non-NAFLD $(n=3849)$ & NAFLD $(n=2859)$ & & Non-NAFLD $(n=784)$ & NAFLD $(n=784)$ & \\
\hline \multicolumn{7}{|l|}{ Clinical characteristics } \\
\hline Age (years) & $42(35-52)$ & $47(39-55)$ & $p \leq 0.01$ & $44(36-53)$ & $47(39-55)$ & $p \leq 0.01$ \\
\hline Gender (male \%) & $2104(54.7)$ & $2162(75.6)$ & $p \leq 0.01$ & $422(53.8)$ & $627(80.0)$ & $p \leq 0.01$ \\
\hline BMI $\left(\mathrm{kg} / \mathrm{m}^{2}\right)$ & $22.9(21.1-24.7)$ & $26.4(24.7-28.3)$ & $p \leq 0.01$ & $22.8(21.1-24.7)$ & $27(25.2-28.9)$ & $p \leq 0.01$ \\
\hline $\begin{array}{l}\text { Systolic pressure } \\
(\mathrm{mmHg})\end{array}$ & $121(111-133)$ & $131(120-143)$ & $p \leq 0.01$ & $121(112-134)$ & $131(121-142)$ & $p \leq 0.01$ \\
\hline $\begin{array}{l}\text { Diastolic pressure } \\
(\mathrm{mmHg})\end{array}$ & $74(66-82)$ & $81(73-89)$ & $p \leq 0.01$ & $74(67-82)$ & $82(75-90)$ & $p \leq 0.01$ \\
\hline Hypertension (\%) & $735(19.1)$ & $1009(32.3)$ & $p \leq 0.01$ & $176(22.4)$ & $293(37.3)$ & $p \leq 0.01$ \\
\hline Diabetes (\%) & $88(2.3)$ & $259(9.1)$ & $p \leq 0.01$ & $24(3.1)$ & $121(15.4)$ & $p \leq 0.01$ \\
\hline Metabolic syndrome (\%) & $221(5.7)$ & $733(25.6)$ & $p \leq 0.01$ & $46(5.9)$ & $84(10.7)$ & $p \leq 0.01$ \\
\hline \multicolumn{7}{|l|}{ Biochemical data } \\
\hline $\mathrm{GCA}(\mu \mathrm{g} / \mathrm{mL})$ & $1.28(1.08-1.50)$ & $1.30(1.10-1.53)$ & $p \leq 0.01$ & $1.27(1.05-1.52)$ & $1.43(1.25-1.65)$ & $p \leq 0.01$ \\
\hline TBA $(\mu \mathrm{mol} / \mathrm{L})$ & $2.5(1.8-3.7)$ & $2.8(2.0-4.2)$ & $p \leq 0.01$ & $2.5(1.8-4.0)$ & $3.0(2.1-4.4)$ & $p \leq 0.01$ \\
\hline GCA-to-TBA ratio & $0.48(0.36-0.64)$ & $0.44(0.33-0.60)$ & $p \leq 0.01$ & $1.02(0.78-1.35)$ & $0.47(0.36-0.65)$ & $p \leq 0.01$ \\
\hline $\operatorname{ALT}(\mathrm{IU} / \mathrm{L})$ & $17(13-24)$ & $28(20-42)$ & $p \leq 0.01$ & $17(12-24)$ & $31(22-47)$ & $p \leq 0.01$ \\
\hline AST (IU/L) & $21(18-24)$ & $24(20-30)$ & $p \leq 0.01$ & $20(17-24)$ & $26(22-32)$ & $p \leq 0.01$ \\
\hline ALP (IU/L) & $69(57-84)$ & $76(64-89)$ & $p \leq 0.01$ & $69(57-84)$ & $77(66.7-90)$ & $p \leq 0.01$ \\
\hline$\gamma$-GT $(\mathrm{IU} / \mathrm{L})$ & $18(14-28)$ & $33(22-54)$ & $p \leq 0.01$ & $18(14-27)$ & $38(25-63.25)$ & $p \leq 0.01$ \\
\hline BUN (mmol/L) & $4.9(4.2-5.8)$ & $5.1(4.4-5.9)$ & $p \leq 0.01$ & $4.9(4.2-5.8)$ & $5.2(4.4-5.9)$ & $p \leq 0.01$ \\
\hline Creatinine $(\mu \mathrm{mol} / \mathrm{L})$ & $77(66-87)$ & $81(72-89)$ & $p \leq 0.01$ & $76(65.5-87)$ & $83(74-91)$ & $p \leq 0.01$ \\
\hline Uric acid $(\mu \mathrm{mol} / \mathrm{L})$ & $322(266-386)$ & $387(331-448)$ & $p \leq 0.01$ & $318.3(263-383)$ & $399(347-459)$ & $p \leq 0.01$ \\
\hline $\mathrm{FBG}(\mathrm{mmol} / \mathrm{L})$ & $4.93(4.49-5.23)$ & $5.21(4.89-5.70)$ & $p \leq 0.01$ & $4.94(4.66-5.29)$ & $5.38(4.99-6.00)$ & $p \leq 0.01$ \\
\hline Triglyceride (mmol/L) & $1.08(0.79-1.51)$ & $1.81(1.3-2.56)$ & $p \leq 0.01$ & $1.09(0.80-1.50)$ & $1.97(1.40-2.70)$ & $p \leq 0.01$ \\
\hline $\begin{array}{l}\text { Total cholesterol } \\
(\mathrm{mmol} / \mathrm{L})\end{array}$ & $4.86(4.27-5.50)$ & $5.19(4.59-5.83)$ & $p \leq 0.01$ & $4.88(4.26-5.54)$ & $5.25(4.65-5.88)$ & $p \leq 0.01$ \\
\hline $\mathrm{HDL}-\mathrm{C}(\mathrm{mmol} / \mathrm{L})$ & $1.29(1.11-1.49)$ & $1.11(0.99-1.28)$ & $p \leq 0.01$ & $1.30(1.12-1.50)$ & $1.08(0.96-1.11)$ & $p \leq 0.01$ \\
\hline $\mathrm{LDL}-\mathrm{C}(\mathrm{mmol} / \mathrm{L})$ & $3.09(2.65-3.59)$ & $3.42(2.98-3.90)$ & $p \leq 0.01$ & $3.09(2.63-3.62)$ & $3.45(3.01-3.92)$ & $p \leq 0.01$ \\
\hline $\mathrm{FFA}(\mu \mathrm{mol} / \mathrm{L})$ & $0.42(0.31-0.52)$ & $0.44(0.35-0.56)$ & $p \leq 0.01$ & $0.43(0.32-0.53)$ & $0.45(0.37-0.57)$ & $p \leq 0.01$ \\
\hline
\end{tabular}

Data are shown as the median and interquartile range (IQR) or percentages. $p$ values were from the analysis of the Wilcoxon rank sum test or Pearson's $x^{2}$ test. A two-tailed $p$ value of less than 0.05 was considered statistically significant. NAFLD: nonalcoholic fatty liver disease; BMI: body mass index; GCA: glycocholic acid; TBA: total bile acids; ALT: alanine aminotransferase; AST: aspartate aminotransferase; ALP: alkaline phosphatase; $\gamma$-GT: $\gamma$-glutamyl transpeptidase; BUN: blood urea nitrogen; FBG: fasting blood glucose; LDL-C: low-density lipoprotein cholesterol; HDL-C: high-density lipoprotein cholesterol; FFA: free fatty acid.

0.55 (95\% CI: 0.53-0.57) with a cutoff value of 0.4 , and the sensitivity and specificity were 0.53 and 0.55 , respectively, which suggested that the GCA-to-TBA ratio alone could not discriminate NAFLD well.

In order to improve the ability of the GCA-to-TBA ratio in discriminating NAFLD, a novel discriminating model (GCA-to-TBA ratio score model) incorporating the GCAto-TBA ratio and other variables with $p<0.05$ in multivariate logistic analysis (age, gender, BMI, diabetes, and metabolic syndrome). The developed novel model is shown as follows: $\quad$ GCA-to-TBA ratio score model $=-12.0-0.21 \times$ GCA-to-TBA ratio $+0.44 \times$ BMI $+0.02 \times$ age $+0.90 \times$ MTS $+0.38 \times$ gender $+0.50 \times \mathrm{DM}$. The ROC of the GCA-to-TBA ratio score model for discriminating NAFLD was 0.84 (95\% CI: $0.83-0.85$ ), and the sensitivity and specificity were $80 \%$ and $72 \%$, respectively. The nomogram of the GCA-to-TBA ratio score model for discriminating NAFLD is shown in Figure 3. The discriminating performance of the GCA-toTBA ratio score was compared with that of two commonly used models: the HSI and TyG. The AUROC of HSI for discriminating NAFLD was 0.83 (95\% CI: 0.827-0.846) with $80 \%$ sensitivity and $72 \%$ specificity, and the TyG index discriminating NAFLD had an AUROC of 0.78 (95\% CI: $0.769-0.791$ ) with $75 \%$ sensitivity and $68 \%$ specificity. Results of the DeLong test showed that the performance of the developed novel model was superior to that of the TyG index 


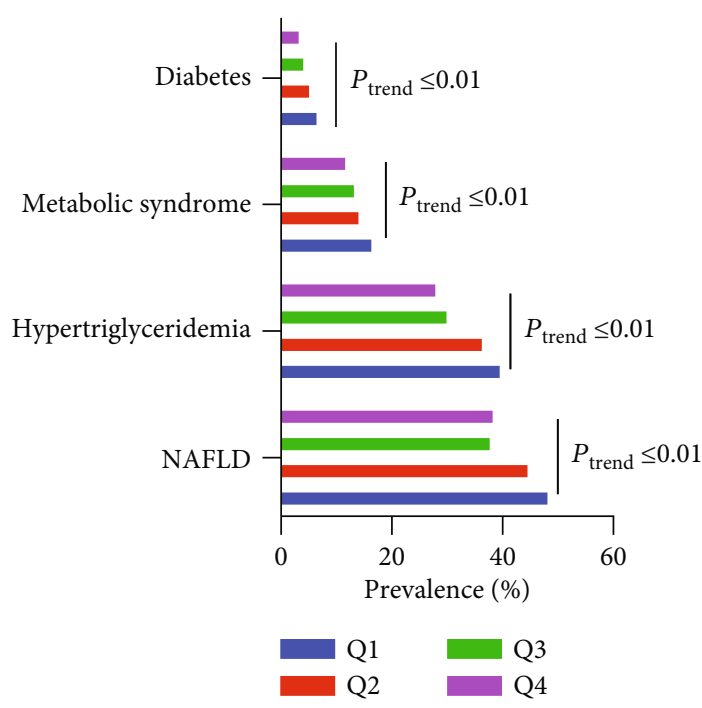

Figure 1: Prevalence of NAFLD, hypertriglyceridemia, metabolic syndrome, and diabetes with different quartiles of the GCA-toTBA ratio. The incidences of NAFLD, metabolic syndrome, hypertriglyceridemia, and diabetes decrease with the increase in the GCA-to-TBA ratio. NAFLD: nonalcoholic fatty liver disease; GCA: glycocholic acid; TBA: total bile acids.

$(p \leq 0.01)$ and noninferior to that of HSI $(p=0.252)$ for discriminating NAFLD from the general population (Figure 4(a)).

3.6. Validation of the GCA-to-TBA Ratio Score Model. An independent validation cohort consisting of 1568 subjects (784 cases and 784 controls) was used to validate the GCAto-TBA ratio score model. The median values of TBA and GCA in the subjects with NAFLD were also significantly higher than those in the subjects without NAFLD (3.0 (2.1$4.4)$ vs. $2.5(1.8-4.0)(p \leq 0.01)$ and $1.43(1.25-1.65)$ vs. 1.27 (1.05-1.52) ( $p \leq 0.01)$, respectively). The GCA-to-TBA ratio in the subjects with NAFLD was significantly lower than that in the subjects without NAFLD (0.47 (0.36-0.65) vs 1.02 (0.78-1.35), $p \leq 0.01)$. Other clinical characteristics and biochemical data are listed in Table 1. In the validation cohort, the AUROC of the GCA-to-TBA ratio score model for discriminating NAFLD was 0.91 (95\% CI: 0.89-0.92), which was better than that of the HSI 0.83 (95\% CI: 0.81-0.85) and TyG index 0.58 (95\% CI: 0.56-0.61) detected with the DeLong test $(p \leq 0.01)$ (Figure $4(\mathrm{~b}))$.

\section{Discussion}

A better understanding of the role of BAs in the development of NAFLD contributes to identifying new biomarkers or therapeutic targets for NAFLD. In this study, we found that the serum GCA-to-TBA ratio was significantly lower in subjects with NAFLD than those without NAFLD. Multivariate logistic regression analysis showed that the GCA-to-TBA ratio was independently associated with NAFLD development after adjusting for age, sex, BMI, diabetes, hypertension, and metabolic syndrome. These results were further confirmed in the sex-stratified analysis. In spite of the fact that the GCA-to-TBA ratio alone could not discriminate
NAFLD from the general population well, a developed novel model incorporating the GCA-to-TBA ratio and other variables had a good performance. Furthermore, we also confirmed that several known risk factors, such as older age, higher BMI, diabetes, and presence of metabolic syndrome, were closely associated with NAFLD, which was consistent with results of previous studies [21-23].

In a recent large-scale study conducted by Zhang et al. [14], which involved 152,336 participants, $27.5 \%$ of the study population had NAFLD. The level of serum TBA in patients with NAFLD was significantly higher than that in those without NAFLD (3.4 vs. $3.0 \mu \mathrm{mol} / \mathrm{L}, p \leq 0.01$ ). However, serum TBA level was not independently associated with NAFLD in multivariate regression analysis. Although the sample size in our study was smaller than that in Zhang et al.'s study, our results further confirmed the similar result of elevated serum TBA level in subjects with NAFLD. Moreover, our study also found that the GCA level in subjects with NAFLD was significantly higher than that in subjects without NAFLD, but neither serum GCA nor TBA level was associated with NAFLD in multivariate analysis. Interestingly and most importantly, we found that the GCA-to-TBA ratio was significantly lower in subjects with NAFLD compared with those without NAFLD and independently associated with NAFLD.

Serum BAs are a diverse collection of amphipathic steroid molecules, including primary BAs, secondary BAs, and their conjugates. Chenodeoxycholic acid (CDCA) and cholic acid (CA) are two major types of primary BAs in the serum. When secreted into the intestine from the gall bladder, CDCA and CA are transformed into lithocholic acid (LCA) and deoxycholic acid (DCA) by gut microbiota, respectively, which are the major types of secondary BAs in the serum [24]. Most of the BAs in the intestine are reabsorbed into the portal vein and act directly on BA receptors in the liver, such as FXR and TGR5 [25]. These BAs play an essential role in regulating glucose and lipid metabolism and maintaining energy homeostasis. However, different types of BAs have distinct regulatory effects on glucose and lipid metabolism. CDCA is the most potent agonist of FXR compared with other BAs, whereas DCA and LCA function as FXR antagonists. The complex functions of different types of BAs might partly explain the lack of association between systemic TBAs and NAFLD. Although the systemic BA composition is quite different from that in the portal blood, systemic BAs could be used as biomarkers for identifying NAFLD. Comparison between the BA composition in the peripheral blood and in the portal blood warrants further research.

Previous studies reported inconsistent results with regard to BA metabolism in the development of NAFLD. In a recent study [9] that enrolled 16 patients with biopsy-proven NASH and 11 healthy controls, the serum TBA levels in patients with NASH were over three times higher than those in healthy controls, and the absolute concentrations of both primary and secondary BAs were elevated in patients with NASH. However, the percentage of CDCA, the major primary BAs in serum, was decreased in patients with NASH compared with healthy controls. As CDCA is the most potent FXR agonist compared with other BAs, a decreased percentage of CDCA may indicate a suppressed FXR signaling, 
TABLE 2: Basic characteristics of the study population categorized by quartiles of the GCA-to-TBA ratio.

\begin{tabular}{|c|c|c|c|c|c|}
\hline Parameters & Q1 $(n=1677)$ & Q2 $(n=1684)$ & Q3 $(n=1691)$ & $\mathrm{Q} 4(n=1656)$ & $p$ \\
\hline \multicolumn{6}{|l|}{ Clinical characteristics } \\
\hline Age (years) & $46(37-55)$ & $45(36-54)$ & $43(36-52)$ & $44(36-53)$ & $p \leq 0.01$ \\
\hline Gender, male & $1186(70.7)$ & $1123(66.7)$ & $1036(61.3)$ & $921(55.6)$ & $p \leq 0.01$ \\
\hline BMI (kg/m2) & $24.8(22.6-27.1)$ & $24.6(22.4-26.8)$ & $24.1(22.1-26.5)$ & $24.1(22.0-26.6)$ & $p \leq 0.01$ \\
\hline NAFLD (\%) & $815(48.6)$ & $757(45.0)$ & $646(38.2)$ & $641(38.7)$ & $p \leq 0.01$ \\
\hline Diabetes (\%) & $116(6.9)$ & $94(5.6)$ & $76(4.5)$ & $61(3.7)$ & $p \leq 0.01$ \\
\hline Metabolic syndrome (\%) & $282(16.8)$ & $244(14.5)$ & $232(13.7)$ & $200(12.1)$ & $p \leq 0.01$ \\
\hline Hypertension (\%) & $525(31.3)$ & $445(26.4)$ & $421(24.9)$ & $373(22.5)$ & $p \leq 0.01$ \\
\hline \multicolumn{6}{|l|}{ Biochemical data } \\
\hline ALT (IU/L) & $23(16-35)$ & $22(15-34)$ & $20(14-30)$ & $19(14-28)$ & $p \leq 0.01$ \\
\hline AST (IU/L) & $23(19-28)$ & $22(19-27)$ & $21(18-26)$ & $21(18-25)$ & $p \leq 0.01$ \\
\hline $\operatorname{ALP}(\mathrm{IU} / \mathrm{L})$ & $75(62-89)$ & $73(61-86)$ & $71(59-85)$ & $70(59-85)$ & $p \leq 0.01$ \\
\hline$\gamma$-GT $(\mathrm{IU} / \mathrm{L})$ & $27(17-44)$ & $25(16-42)$ & $22(15-36)$ & $21(15-34)$ & $p \leq 0.01$ \\
\hline $\mathrm{BUN}(\mathrm{mmol} / \mathrm{L})$ & $5.0(4.3-5.9)$ & $5.1(4.3-6.0)$ & $5.0(4.3-5.8)$ & $4.9(4.2-5.7)$ & $p \leq 0.01$ \\
\hline Creatinine $(\mu \mathrm{mol} / \mathrm{L})$ & $80(71-88)$ & $80(68-88)$ & $78(68-88)$ & $78(66-88)$ & $p \leq 0.01$ \\
\hline Uric acid $(\mu \mathrm{mol} / \mathrm{L})$ & $359(299-425)$ & $358(292-422)$ & $348(285-411)$ & $340(279-406)$ & $p \leq 0.01$ \\
\hline $\mathrm{FBG}(\mathrm{mmol} / \mathrm{L})$ & $5.10(4.76-5.50)$ & $5.04(4.75-5.46)$ & $5.05(4.73-5.40)$ & $5.01(4.73-5.38)$ & $p \leq 0.01$ \\
\hline Triglyceride (mmol/L) & $1.48(1.02-2.23)$ & $1.37(0.94-2.09)$ & $1.27(0.88-1.88)$ & $1.25(0.89-1.79)$ & $p \leq 0.01$ \\
\hline Total cholesterol (mmol/L) & $4.98(4.41-5.68)$ & $5.04(4.38-5.67)$ & $5.00(4.39-5.64)$ & $4.96(4.37-5.60)$ & 0.312 \\
\hline HDL-C (mmol/L) & $1.17(1.02-1.36)$ & $1.20(1.05-1.41)$ & $1.22(1.06-1.43)$ & $1.23(1.06-1.42)$ & $p \leq 0.01$ \\
\hline LDL-C (mmol/L) & $3.24(2.80-3.74)$ & $3.25(2.75-3.77)$ & $3.23(2.76-3.73)$ & $3.21(2.74-3.70)$ & 0.225 \\
\hline
\end{tabular}

Data are shown as the median and interquartile range (IQR) or percentages. $p$ values were from analysis of the Kruskal-Wallis test or Pearson's $x^{2}$ test. A twotailed $p$ value of less than 0.05 was considered statistically significant. NAFLD: nonalcoholic fatty liver disease; BMI: body mass index; ALT: alanine aminotransferase; AST: aspartate aminotransferase; ALP: alkaline phosphatase; $\gamma$-GT: $\gamma$-glutamyl transpeptidase; TBA: total bile acids; GCA: glycocholic acid; BUN: blood urea nitrogen; FBG: fasting blood glucose; LDL-C: low-density lipoprotein cholesterol; HDL-C: high-density lipoprotein cholesterol; Q1: the GCA-to-TBA ratio < 0.75; Q2: the GCA-to-TBA ratio 0.75-1.00; Q3: the GCA-to-TBA ratio 1.00-1.34; Q4: the GCA-to-TBA ratio $\geq 1.3$.

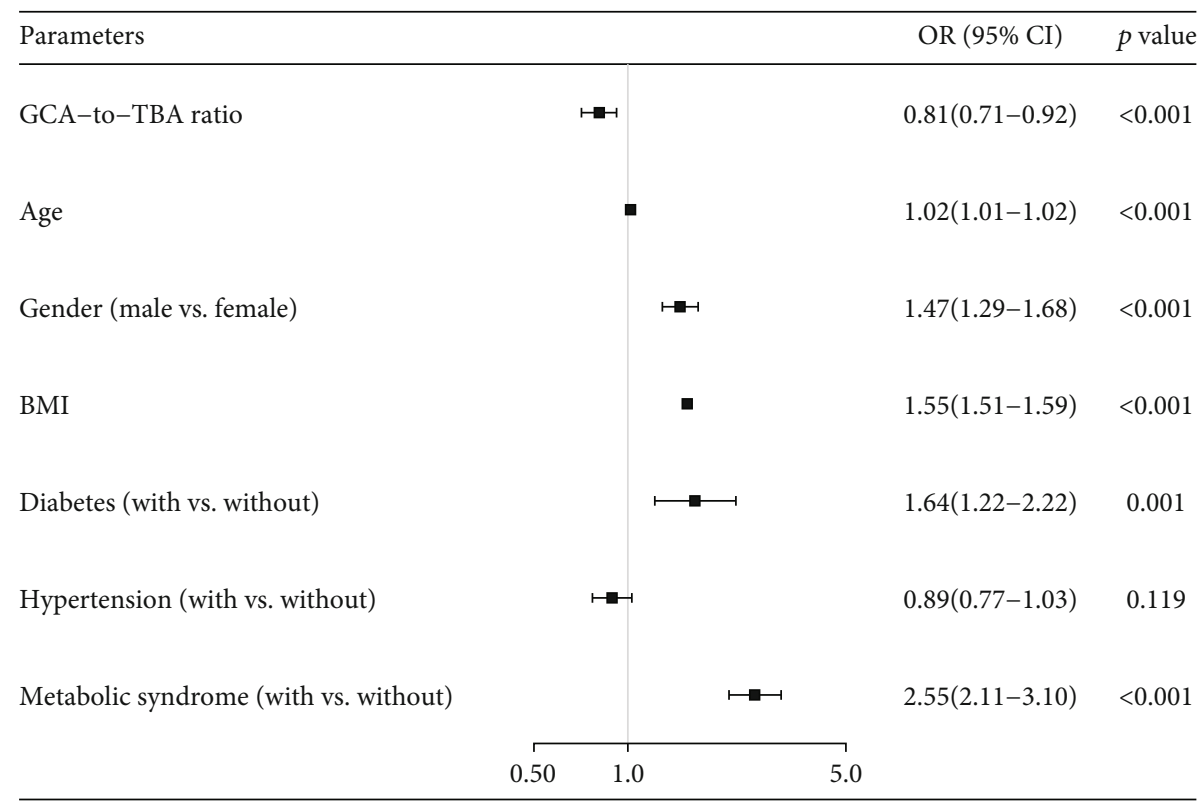

FIGURE 2: The GCA-to-TBA ratio is independently associated with NAFLD development. OR: odds ratio; CI: confidence intervals; GCA: glycocholic acid; TBA: total bile acids. 
TABLE 3: GCA-to-TBA ratio was independently associated with NAFLD in both male and female individuals.

\begin{tabular}{|c|c|c|c|c|c|c|}
\hline \multirow{2}{*}{ Parameters } & \multicolumn{3}{|c|}{ Male } & \multicolumn{3}{|c|}{ Female } \\
\hline & OR & $95 \% \mathrm{CI}$ & $p$ & OR & $95 \% \mathrm{CI}$ & $p$ \\
\hline GCA-to-TBA ratio & 0.85 & $0.73-0.99$ & 0.042 & 0.73 & $0.58-0.92$ & $p \leq 0.01$ \\
\hline Age & 1.01 & $1.00-1.02$ & $p \leq 0.01$ & 1.04 & $1.03-1.05$ & $p \leq 0.01$ \\
\hline BMI & 1.51 & $1.46-1.56$ & $p \leq 0.01$ & 1.64 & $1.56-1.72$ & $p \leq 0.01$ \\
\hline Diabetes (with vs. without) & 1.62 & $1.17-2.27$ & $p \leq 0.01$ & 2.11 & $1.03-4.47$ & 0.046 \\
\hline Hypertension (with vs. without) & 0.96 & $0.81-1.14$ & 0.667 & 0.64 & $0.47-0.86$ & $p \leq 0.01$ \\
\hline Metabolic syndrome (with vs. without) & 2.22 & $1.77-2.78$ & $p \leq 0.01$ & 3.60 & $2.48-5.27$ & $p \leq 0.01$ \\
\hline
\end{tabular}

NAFLD: nonalcoholic fatty liver disease; TBA: total bile acids; GCA: glycocholic acid; BMI: body mass index; OR: odds ratio; CI: confidence interval.

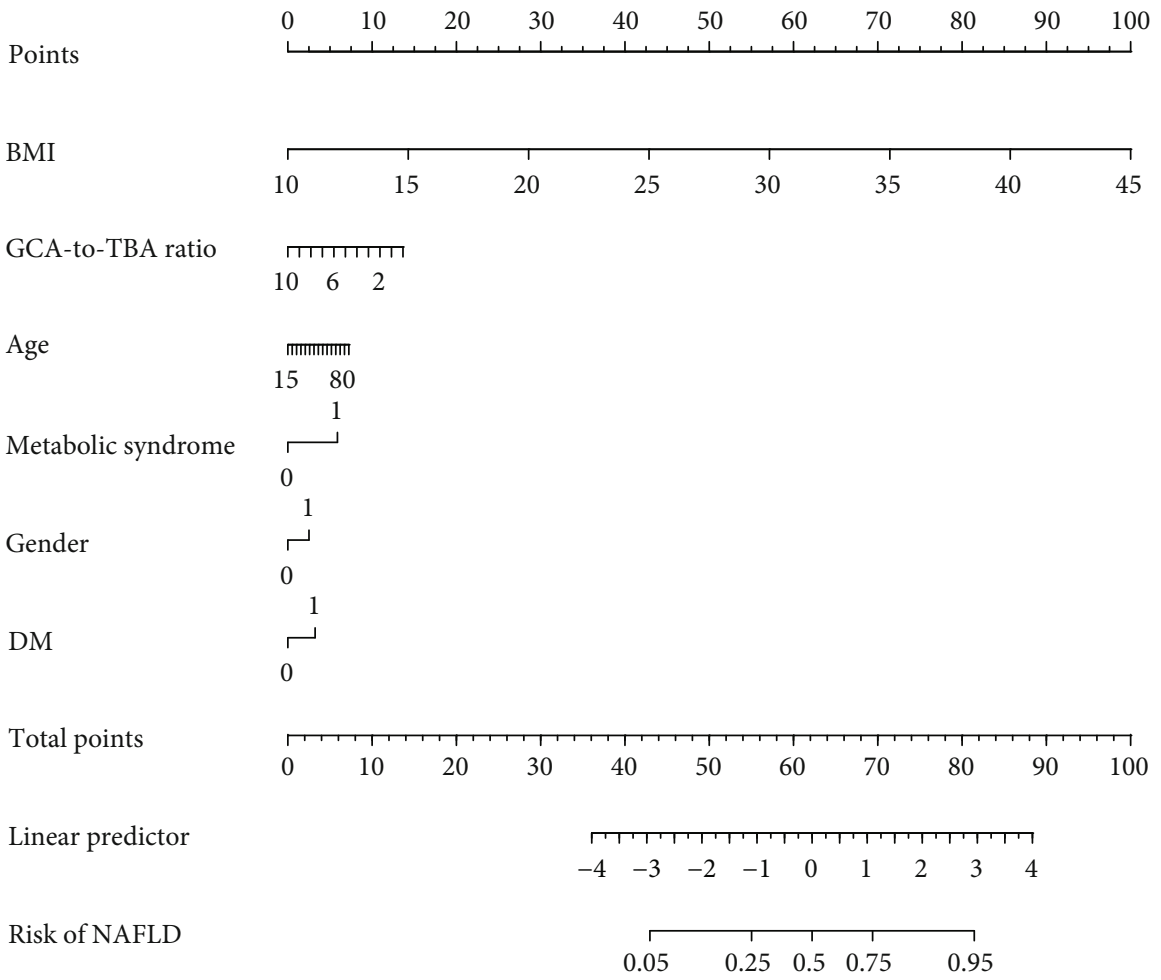

FIGURE 3: Nomogram of the GCA-to-TBA ratio score model for discriminating NAFLD for the general papulation. Metabolic syndrome $=1$ indicating subjects with metabolic syndrome, gender $=1$ indicating male subjects, and $\mathrm{DM}=1$ indicating subjects with diabetes. GCA: glycocholic acid; TBA: total bile acids; BMI: body mass index; DM: diabetes.

which was supported by a reduced serum fibroblast growth factor 19 level in patients with NASH. In our study, patients with NAFLD had increased absolute concentrations of serum TBA and GCA but decreased percentage of GCA, which also supported the suppressed FXR signaling in patients with NAFLD indirectly, although GCA is a less potent agonist for FXR compared with CDCA [26]. However, Makishima et al. [26] showed in an animal study that increased serum BA levels in high-fat diet-induced obese mice were correlated with weight loss after vertical sleeve gastrectomy, and the BAs and their receptors, FXR or TGR5, served as important molecules for the beneficial effects of this weight loss surgery. By contrast, in a study carried out by Puri et al. [13], patients with NAFLD had significantly elevated plasma primary BAs but lower plasma secondary BAs compared with healthy controls. The reason for this discrepancy may be due to the different designs of the two studies, but changes in the intestinal microbiota in patients with $\mathrm{NASH}$, which would reduce the formation of secondary BAs, might partly explain the decreased secondary BAs in NAFLD.

The present study has two limitations. First, other types of serum BAs, such as CDCA, were not detected in the present study. Alterations of serum BA profiles may generally provide more information about metabolic homeostasis. However, the unique detection approach and higher cost limit their clinical applications. By comparison, the serum TBA and GCA are more widely detected in the clinic, which might provide a more practical and convenient option for 


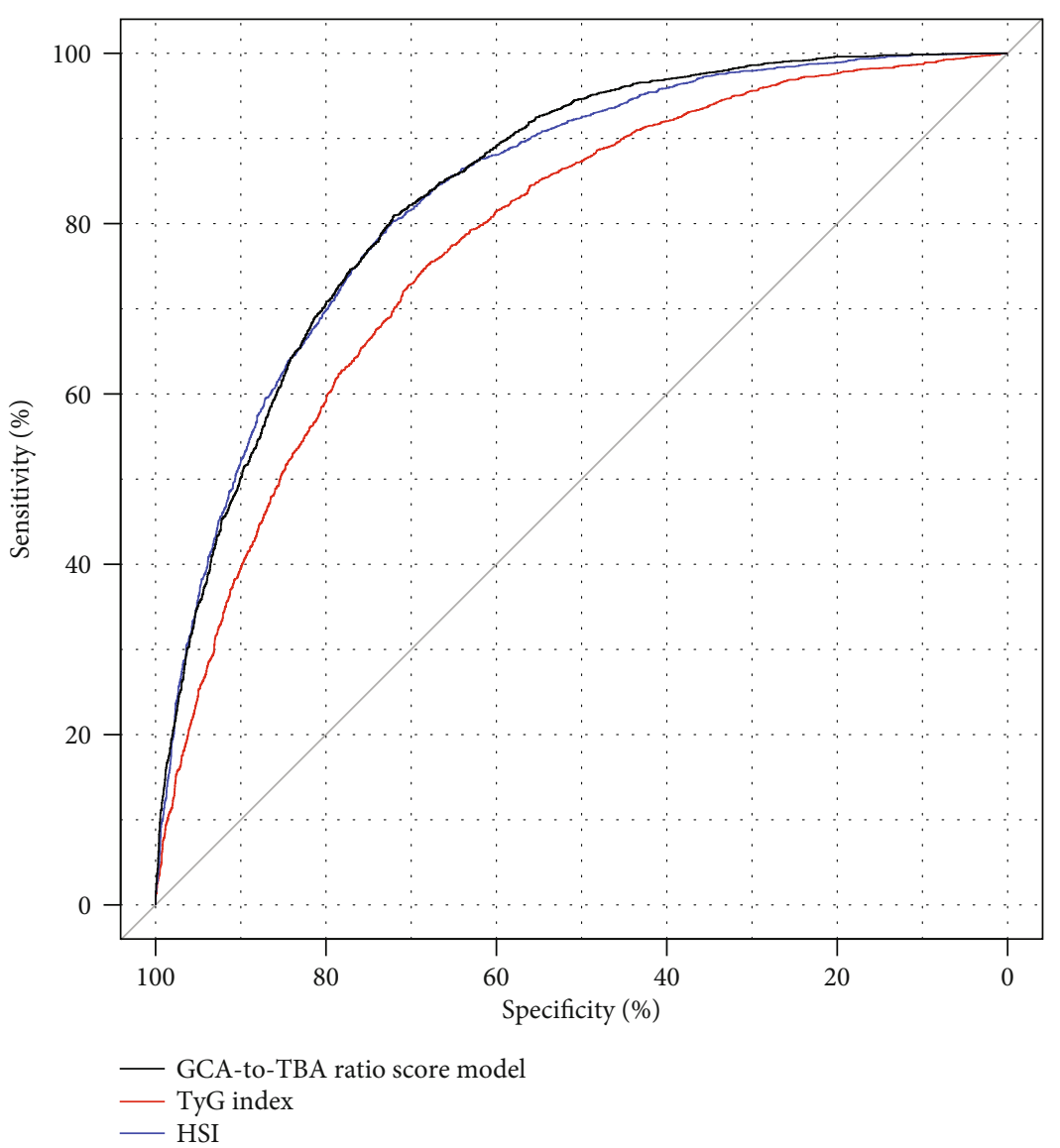

(a)

Figure 4: Continued. 


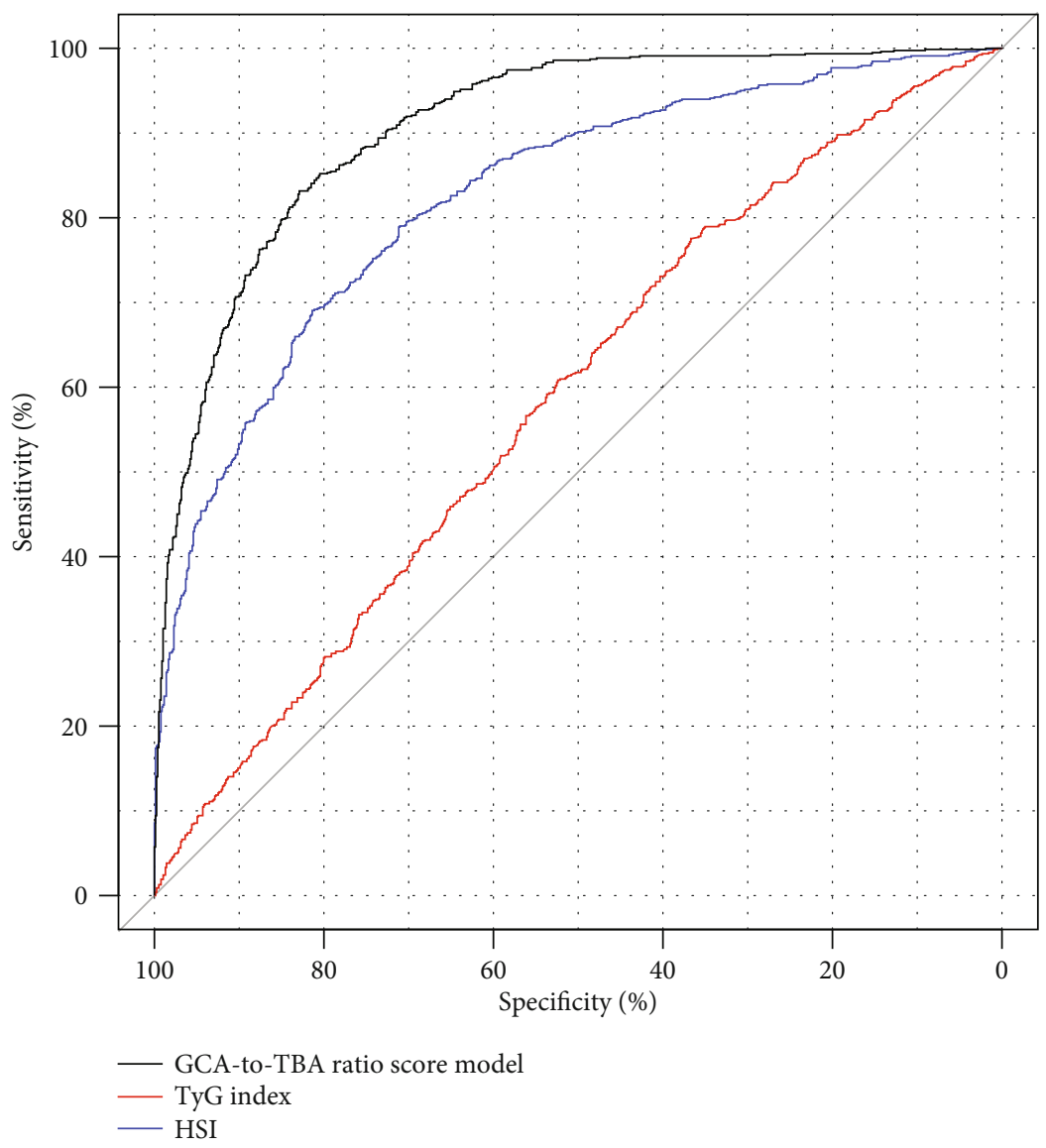

(b)

FIgure 4: Comparison of the HSI, TyG index, and GCA-to-TBA ratio score model for predicting the performance of NAFLD in the development (a) and validation cohort (b). Black line indicating the GCA-to-TBA ratio score model, blue line indicating the HSI, and red line indicating the TyG index. NAFLD: nonalcoholic fatty liver disease; HSI: hepatic steatosis index; TyG index: triglyceride $\times$ glucose index; GCA: glycocholic acid; TBA: total bile acids.

predicting NAFLD. Second, although our study shows that a decreased GCA-to-TBA ratio indicates higher likelihood of suffering from NAFLD, ultrasonography is still a convenient tool for diagnosing NAFLD. If the serum GCA-to-TBA ratio could act as a biomarker for identifying NASH from NAFLD patients, it would make more sense but need further study in biopsy-proven NAFLD patients. Nevertheless, our primary data demonstrated that the GCA-to-TBA ratio was independently associated with NAFLD, and a novel model incorporating the GCA-to-TBA ratio had a good performance in screening NAFLD from the general population. Therefore, as signal molecules for energy homeostasis, the GCA-toTBA ratio will draw more attention to other metabolic disorders.

\section{Conclusions}

Taken together, our data suggested that the GCA-to-TBA ratio was lower in subjects with NAFLD than in those without NAFLD and was independently associated with NAFLD development. A novel model incorporating the GCA-to-TBA ratio may serve as a simple tool for identifying NAFLD from the general population in the clinics.

\section{Data Availability}

The data of this study are available from the corresponding authors upon reasonable request.

\section{Conflicts of Interest}

The authors declare that they have no competing interests.

\section{Authors' Contributions}

$\mathrm{Hu} \mathrm{Li}$ and Jin Ma contributed equally to this work.

\section{Acknowledgments}

We thank all the staff in the Clinical Laboratory Diagnostic Center for their technical assistance and all the participants enrolled in the study. This work was supported in part by the Ruijin Hospital Research Initiative Project (grant number 2019ZX01) and Jiading Science and Technology Commission Project (grant number JDKW-2018-W10). 


\section{References}

[1] J. Li, B. Zou, Y. H. Yeo et al., "Prevalence, incidence, and outcome of non-alcoholic fatty liver disease in Asia, 1999-2019: a systematic review and meta-analysis," The Lancet Gastroenterology \& Hepatology, vol. 4, no. 5, pp. 389-398, 2019.

[2] Z. M. Younossi, A. B. Koenig, D. Abdelatif, Y. Fazel, L. Henry, and M. Wymer, "Global epidemiology of nonalcoholic fatty liver disease-meta-analytic assessment of prevalence, incidence, and outcomes," Hepatology, vol. 64, no. 1, pp. 73-84, 2016.

[3] Z. M. Younossi, P. Golabi, L. de Avila et al., "The global epidemiology of NAFLD and NASH in patients with type 2 diabetes: a systematic review and meta-analysis," Journal of Hepatology, vol. 71, no. 4, pp. 793-801, 2019.

[4] H. L. Zhai, N. J. Wang, B. Han et al., "Low vitamin D levels and non-alcoholic fatty liver disease, evidence for their independent association in men in East China: a cross-sectional study (Survey on Prevalence in East China for Metabolic Diseases and Risk Factors (SPECT-China))," The British Journal of Nutrition, vol. 115, no. 8, pp. 1352-1359, 2016.

[5] Z. Younossi, F. Tacke, M. Arrese et al., "Global perspectives on nonalcoholic fatty liver disease and nonalcoholic steatohepatitis," Hepatology, vol. 69, no. 6, pp. 2672-2682, 2019.

[6] M. E. Rinella, "Nonalcoholic fatty liver disease: a systematic review," JAMA, vol. 313, no. 22, pp. 2263-2273, 2015.

[7] A. Molinaro, A. Wahlström, and H. U. Marschall, "Role of bile acids in metabolic control," Trends in Endocrinology and Metabolism, vol. 29, no. 1, pp. 31-41, 2018.

[8] J. P. Arab, S. J. Karpen, P. A. Dawson, M. Arrese, and M. Trauner, "Bile acids and nonalcoholic fatty liver disease: molecular insights and therapeutic perspectives," Hepatology, vol. 65, no. 1, pp. 350-362, 2017.

[9] N. Jiao, S. S. Baker, A. Chapa-Rodriguez et al., "Suppressed hepatic bile acid signalling despite elevated production of primary and secondary bile acids in NAFLD," Gut, vol. 67, no. 10, pp. 1881-1891, 2018.

[10] B. C. Ferslew, G. Xie, C. K. Johnston et al., "Altered bile acid metabolome in patients with nonalcoholic steatohepatitis," Digestive Diseases and Sciences, vol. 60, no. 11, pp. 33183328, 2015.

[11] B. C. Ferslew, C. K. Johnston, E. Tsakalozou et al., “Altered morphine glucuronide and bile acid disposition in patients with nonalcoholic steatohepatitis," Clinical Pharmacology and Therapeutics, vol. 97, no. 4, pp. 419-427, 2015.

[12] C. Caussy, C. Hsu, S. Singh et al., "Serum bile acid patterns are associated with the presence of NAFLD in twins, and dosedependent changes with increase in fibrosis stage in patients with biopsy-proven NAFLD," Alimentary Pharmacology \& Therapeutics, vol. 49, no. 2, pp. 183-193, 2019.

[13] P. Puri, K. Daita, A. Joyce et al., "The presence and severity of nonalcoholic steatohepatitis is associated with specific changes in circulating bile acids," Hepatology, vol. 67, no. 2, pp. 534$548,2018$.

[14] Z. Zhang, W. Dai, S. Weng et al., "The association of serum total bile acid with non-alcoholic fatty liver disease in Chinese adults: a cross sectional study," Lipids in Health and Disease, vol. 19, no. 1, p. 18, 2020.

[15] L. A. Adams, Z. Wang, C. Liddle et al., "Bile acids associate with specific gut microbiota, low-level alcohol consumption and liver fibrosis in patients with non-alcoholic fatty liver disease," Liver International, vol. 40, no. 6, pp. 1356-1365, 2020.
[16] N. Chalasani, Z. Younossi, J. E. Lavine et al., "The diagnosis and management of nonalcoholic fatty liver disease: practice guidance from the American Association for the Study of Liver Diseases," Hepatology, vol. 67, no. 1, pp. 328-357, 2018.

[17] J. B. Buse, D. J. Wexler, A. Tsapas et al., "2019 update to: management of hyperglycemia in type 2 diabetes, 2018. A consensus report by the American Diabetes Association (ADA) and the European Association for the Study of Diabetes (EASD)," Diabetes Care, vol. 43, no. 2, pp. 487-493, 2020.

[18] J. H. Lee, D. Kim, H. J. Kim et al., "Hepatic steatosis index: a simple screening tool reflecting nonalcoholic fatty liver disease," Digestive and Liver Disease, vol. 42, no. 7, pp. 503-508, 2010.

[19] F. Guerrero-Romero, L. E. Simental-Mendía, M. GonzálezOrtiz et al., "The product of triglycerides and glucose, a simple measure of insulin sensitivity. Comparison with the euglycemic-hyperinsulinemic clamp," The Journal of Clinical Endocrinology \& Metabolism, vol. 95, no. 7, pp. 3347-3351, 2010.

[20] X. Robin, N. Turck, A. Hainard et al., "pROC: an open-source package for R and S+ to analyze and compare ROC curves," BMC Bioinformatics, vol. 12, no. 1, p. 77, 2011.

[21] A. Sasaki, H. Nitta, K. Otsuka et al., "Bariatric surgery and non-alcoholic fatty liver disease: current and potential future treatments," Frontiers in Endocrinology, vol. 5, p. 164, 2014.

[22] P. Golabi, M. Sayiner, Y. Fazel, A. Koenig, L. Henry, and Z. M. Younossi, "Current complications and challenges in nonalcoholic steatohepatitis screening and diagnosis," Expert Review of Gastroenterology \& Hepatology, vol. 10, no. 1, pp. 63-71, 2016.

[23] N. Fan, L. Zhang, Z. Xia, L. Peng, Y. Wang, and Y. Peng, "Sexspecific association between serum uric acid and nonalcoholic fatty liver disease in type 2 diabetic patients," Journal Diabetes Research, vol. 2016, article 3805372, 6 pages, 2016.

[24] H. Shapiro, A. A. Kolodziejczyk, D. Halstuch, and E. Elinav, "Bile acids in glucose metabolism in health and disease," The Journal of Experimental Medicine, vol. 215, no. 2, pp. 383396, 2018.

[25] B. L. Copple and T. Li, "Pharmacology of bile acid receptors: evolution of bile acids from simple detergents to complex signaling molecules," Pharmacological Research, vol. 104, pp. 921, 2016.

[26] M. Makishima, A. Y. Okamoto, J. J. Repa et al., "Identification of a nuclear receptor for bile acids," Science (New York, N.Y.), vol. 284, no. 5418, pp. 1362-1365, 1999. 